\title{
Real-World Results of Switching Treatment from Ranibizumab to Aflibercept in Macular Oedema Secondary to Branch Retinal Vein Occlusion
}

\author{
Vasileios E. Konidaris (D) - Konstantinos T. Tsaousis • Rossella Anzidei • \\ Guillermo de la Mata · Alexander J. Brent
}

Received: August 17, 2018 / Published online: October 4, 2018

(C) The Author(s) 2018

\section{ABSTRACT}

Introduction: To investigate treatment outcomes after switching from ranibizumab to aflibercept intravitreal injections in patients with macular oedema (MO) secondary to branch retinal vein occlusion (BRVO).

Methods: Eligible patients with refractory MO secondary to BRVO, post treatment with a minimum of three intravitreal injections of ranibizumab at 4 -weekly intervals were recruited. Suboptimal or non-responders were defined as patients who had persistent intraretinal fluid ( $<75 \%$ decrease from baseline) despite a minimum of three consecutive injections. These patients were switched to aflibercept injections on an as-needed basis. The primary study outcomes assessed trends in best-corrected distance visual acuity (BCVA) and central retinal thickness (CRT). To compare means of BCVA and CRT, a paired $t$ test two-tailed with a level of significance set at 0.05 was used. Pearson correlation coefficient was also applied to

Enhanced digital features To view enhanced digital features for this article go to https://doi.org/10.6084/ m9.figshare.7132961.

V. E. Konidaris $(\bowtie) \cdot K$. T. Tsaousis · R. Anzidei · G. de la Mata - A. J. Brent Ophthalmology Department, University Hospitals of Leicester, Leicester Royal Infirmary, Leicester, UK e-mail: vasileios.konidaris@uhl-tr.nhs.uk demonstrate correlation. Participants were followed up for a period of 24 weeks after switching.

Results: Thirty-eight eyes of 38 patients were included in the study. Patients had an average of 8.37 ranibizumab intravitreal injections over a mean period of 12 months presenting suboptimal or no response. A significant decrease of mean CRT from $388.63 \pm 93.4 \mu \mathrm{m}$ to $290.29 \pm 93.5 \mu \mathrm{m}(p<0.001)$ and an improvement in mean BCVA from logMAR $0.66 \pm 0.38$ to $\log$ MAR $0.57 \pm 0.27(p=0.025)$ was achieved after an average of 2.27 aflibercept injections.

Conclusions: Given the spectrum of therapies available to date for the management of MO secondary to BRVO, aflibercept appears to be an effective treatment option in cases refractory to ranibizumab. This study based on a small cohort of patients indicates that satisfactory results on retinal anatomy and visual outcomes can be accomplished with a smaller number of injections. Larger-scale studies are needed to extrapolate these promising results.

Keywords: Aflibercept; Anti-VEGF; Branch retinal vein occlusion; Ranibizumab; Switching

\section{INTRODUCTION}

Branch retinal vein occlusion (BRVO), a relatively common vascular condition affecting the retina, has an incidence of $0.5-1.2 \%$ [1]. It is 
associated with multiple complications such as retinal ischaemia, neovascularisation and macular oedema (MO). MO occurs as a result of several mechanisms and is rarely shown to improve without medical intervention [2]. It is often associated with significant visual loss resulting in debilitating consequences to patients, further emphasising the need for clinicians to identify and manage this group of patients urgently.

Management of patients with MO secondary to BRVO has evolved over the last few decades from the use of grid/sectoral laser as the old gold standard to much newer therapies, involving intravitreal dexamethasone and anti-vascular endothelial growth factors (anti-VEGF). Recent innovative research focusing on the use of these modern therapies such as anti-VEGF therapy to treat $\mathrm{MO}$ has shown particular promise due to overall efficacy. In BRVO with $\mathrm{MO}$, there are a plethora of clinical trials supporting the effectiveness of anti-VEGF therapies aflibercept and ranibizumab. Ranibizumab was the first antiVEGF agent to gain approval in the UK for the management of MO secondary to BRVO in 2013 [3]. Its approval was based on the BRAVO study, which showed that the mean gain in BCVA at 6 months was 18.3 letters with $0.5 \mathrm{mg}$ ranibizumab in comparison to 7.3 letters with the sham injection [4]. On the basis of the VIBRANT study in 2016, aflibercept was licensed for treating MO secondary to BRVO [5]. Despite similarities of the two anti-VEGF agents, the pharmacodynamics of aflibercept and ranibizumab differ greatly. Aflibercept is known to bind with a greater affinity to VEGF resulting in reduced permeability of vessels [6]. It blocks VEGF-induced activation of VEGF receptor 1 and 2 (VEGFR-1/2) with 71 times greater potency than ranibizumab. This added property further supports the rationale of switching antiVEGF treatment in cases demonstrating persisting $\mathrm{MO}$ after ranibizumab treatment. This theoretical and potential benefit in trialling the switching of therapies in BRVO with MO is supported by evidence of success from studies undertaken assessing the benefits of switching anti-VEGF agents in age-related macular degeneration (ARMD), and retinal vascular conditions such as central retinal vein occlusion
(CRVO) with secondary MO and diabetic macular oedema (DMO). In all these retinal conditions, an overall significant improvement was demonstrated in both vision and anatomy of the retina [7-21].

To date, a limited number of studies have published data to assess the effects of switching therapies (anti-VEGF) in patients with BRVO with MO [22-24]. The purpose of this study is to add to this growing evidence of alternative treatments in patients with refractory MO secondary to BRVO, in order to gain a better understanding of the clinical effects of switching treatment from ranibizumab to aflibercept. The study further aims to assess the number of aflibercept injections necessary to achieve resolution of MO.

\section{METHODS}

A prospective audit was conducted in a tertiary retina service at the University Hospitals of Leicester, UK. Ethics approval was not requested, as the data was collected from an audit on current clinical practice (non-interventional) in the Ophthalmology Department of the University Hospitals of Leicester. All procedures performed in studies involving human participants were in accordance with the ethical standards of the institutional and/or national research committee and with the 1964 Helsinki declaration and its later amendments or comparable ethical standards. Informed consent was obtained from all individual participants included in the study.

The following inclusion criteria were applied: all subjects aged at least 18 years (all sexes) diagnosed with BRVO and centre-involving MO (within the five central ETDRS subfields on SD-OCT; 3D OCT-2000) with an ability to understand the purpose of treatment switching.

The exclusion criteria applied included MO not-related to BRVO; known ocular conditions that could affect MO or alter BCVA (e.g. vitreomacular traction; DMO); previous treatment of MO with focal laser; presence of iris/angle/optic disc neovascularisation or elsewhere; neovascular glaucoma; vitreous haemorrhage; previous 
vitrectomy; and uncontrolled glaucoma (greater than $30 \mathrm{mmHg}$ measured with Goldmann applanation tonometer).

At presentation, all patients underwent a thorough systemic and ocular history. This was followed by a thorough ocular examination including intraocular pressure measurement, best corrected distance visual acuity (BCVA) assessment and dilated fundus examination. Patients underwent 4-weekly SD-OCT examinations (3D OCT-2000, Topcon Corporation, Tokyo, Japan) in order to measure the central retinal thickness (CRT) (measured from the thickness map of the OCT platform). Both BCVA and CRT were recorded after every examination visit, at 4-weekly intervals. These changes in CRT recorded were used to assess the response to both types of anti-VEGF treatments and form the basis of the primary outcome of the study.

In patients demonstrating suboptimal response or no response (non-responders) after treatment with a minimum of three ranibizumab $0.5 \mathrm{mg}$ (Lucentis ${ }^{\circledR} 10 \mathrm{mg} / \mathrm{ml}$, Novartis Pharmaceuticals UK Ltd) injections at 4-weekly intervals, switching to aflibercept $2 \mathrm{mg}$ (Eylea ${ }^{\circledR}$ $40 \mathrm{mg} / \mathrm{ml}$, Bayer plc) was performed. A suboptimal response was defined as a combination of a decrease in CRT by $25-75 \%$ from the baseline plus the presence of persisting subretinal or intraretinal fluid. Non-responders were defined as eyes presenting $0-24 \%$ decrease or increase in CRT after the first three monthly ranibizumab injections. These patients underwent switching after the third ranibizumab injection to aflibercept. In cases of suboptimal or non-response, treatment was switched to aflibercept injections on an as-needed basis. The duration of followup after initiation of aflibercept injection was 24 weeks.

Responses to the switched therapy were assessed at 4-weekly intervals until complete resolution of $\mathrm{MO}$ was noted.

All data were collected were analysed using Microsoft Excel 2007 for Windows (Microsoft Corporation, Redmond, WA, USA) and IBM SPSS Statistics software package (SPSS Inc., Chicago, IL, USA). To compare means of VA and CRT, a paired $t$ test two-tailed with a level of significance set at 0.05 was used. Pearson correlation coefficient was also applied to demonstrate correlation.

\section{RESULTS}

The recruitment of patients took place between January and February 2017 and 38 eyes of 38 consecutive patients (19 women) with MO secondary to BRVO with a mean age of $72.9 \pm 9.8(45-88)$ years were studied. Table 1 demonstrates the collated demographic data and clinical characteristics of the 38 patients recruited.

All patients recruited had documented evidence of MO secondary to BRVO refractory to $0.5 \mathrm{mg}$ of intravitreal ranibizumab injections. The persistence of MO despite initial treatment classified their response to be suboptimal. Consequent switching of anti-VEGF treatment to $2 \mathrm{mg} / 0.05 \mathrm{ml}$ intravitreal aflibercept was implemented in lieu of this poor response.

At presentation, the mean CRT of treatment naive patients was $443.84 \pm 98.35 \mu \mathrm{m}$, with a mean BCVA of logMAR $0.65 \pm 0.33$. In a mean

Table 1 Demographics, baseline and follow-up characteristics

\begin{tabular}{lc}
\hline Patients (eyes) & $38(38)$ \\
Mean age, years $( \pm$ SD) & $72.87( \pm 9.84)$ \\
Women, $n(\%)$ & $19(50)$ \\
Previous ranibizumab injections, mean & $8.37(3-17)$ \\
$\quad$ (range) & \\
Aflibercept injections, mean (range) & $2.27(1-3)$ \\
CRT at presentation, mean $( \pm$ SD) & 443.84 \\
& $( \pm 98.35)$ \\
CRT before switch, mean $( \pm$ SD) & 388.63 \\
& $( \pm 93.41)$ \\
CRT after switch, mean $( \pm$ SD) & 290.29 \\
BCVA at presentation, mean $( \pm$ SD) & $0.65( \pm 0.33)$ \\
BCVA before switch, mean $( \pm S D)$ & $0.66( \pm 0.38)$ \\
BCVA after switch, mean $( \pm$ SD $)$ & $0.57( \pm 0.27)$ \\
\hline
\end{tabular}


period of 12 months, a mean number of 8.37 ranibizumab injections were administered without resolution of intraretinal and/or subretinal fluid. The study showed that to achieve complete resolution of $\mathrm{MO}$, with complete absence of intra/subretinal fluid on OCT, an average of 2.27 (1-3) aflibercept injections was administered. It should be noted that complete resolution was successfully achieved in all cases.

At switching, mean CRT was noted as $388.63 \pm 93.41 \mu \mathrm{m}$. After 6 months of aflibercept treatment, a decrease in mean CRT was noted to $290.29 \pm 93.54 \mu \mathrm{m}$ (range $183-358 \mu \mathrm{m})$. As per the primary outcome of the study, a significant mean reduction in CRT of $98.34 \mu \mathrm{m}(p<0.001)$ was noted after switching treatment. It was also noted that the changes in mean BCVA showed a statistically significant improvement from $\operatorname{logMAR}$ $0.66 \pm 0.38$ to $\log$ MAR $0.57 \pm 0.27(p=0.025)$. These findings are further demonstrated in Fig. 1.

This study also noted several other important findings. A statistically significant correlation between CRT and BCVA at presentation (Pearson correlation coefficient $r_{\mathrm{p}}=0.969$ ) was noted. However this correlation was not sustained after switching treatment $\left(r_{\mathrm{p}}=-0.17\right)$ as shown in Fig. 2. The study further demonstrated a statistically significant correlation between

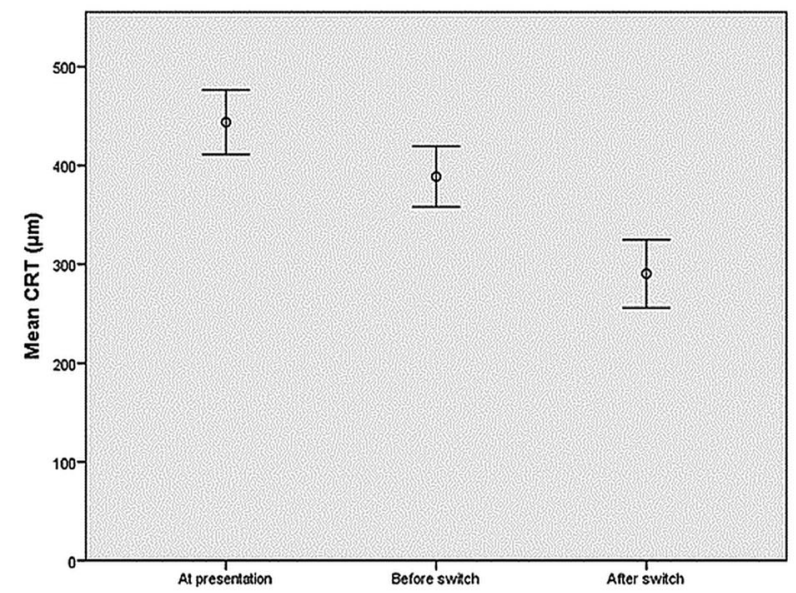

Fig. 1 Change in mean BCVA (a) and CRT (b) at three time points: at presentation, before switching and at 24 weeks after switching treatment to aflibercept. the number of ranibizumab injections with BCVA after switching $\left(r_{\mathrm{p}}=0.374\right)$.

A further subanalysis of the suboptimal and the non-responders to ranibizumab shows that both groups benefited from switching to aflibercept. The non-responders (13 patients) who showed no improvement or even presented with an increase in their CRT during ranibizumab treatment demonstrated a statistically significant decrease in CRT after switching to aflibercept. At switching, mean CRT was noted as $455 \pm 95.74 \mu \mathrm{m}$. After 6 months of aflibercept treatment and following a mean number of injections of 2.36, a decrease in mean CRT was noted to $343.36 \pm 123.97 \mu \mathrm{m}(p=0.008)$. Patients with suboptimal response demonstrated a statistical significant decrease in the CRT after switching treatment to aflibercept from $343.8 \pm 76.1$ to $261.1 \pm 56.6(p<0.001)$.

Throughout the study, all patients were screened for complications associated with the treatment of anti-VEGF therapies. Side effects assessed included endophthalmitis, significantly elevated intraocular pressure, retinal tears or detachments and thromboembolic events. In this study no side effects were reported or noted on follow-up in relation to the intravitreal injections.

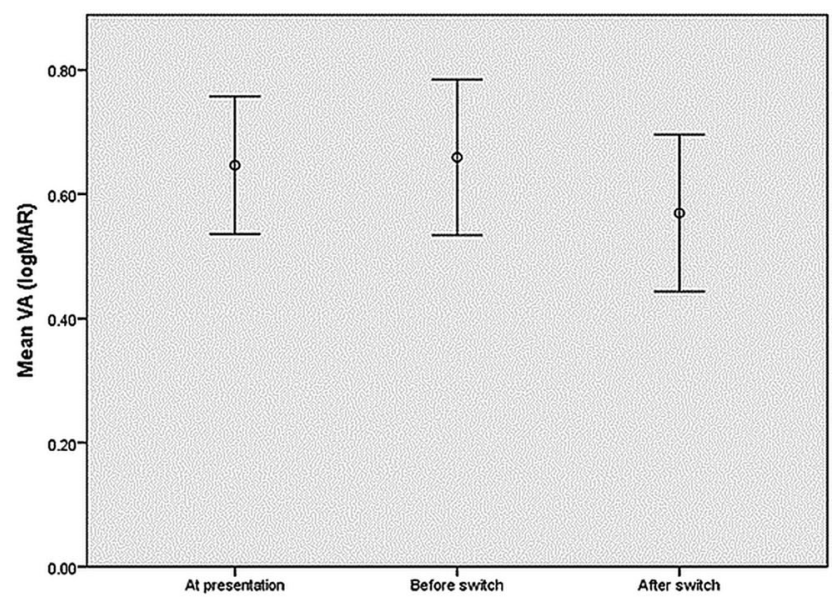

Statistically significant difference in BCVA and CRT was observed between various time points: presentation, before switching and after switching treatment 


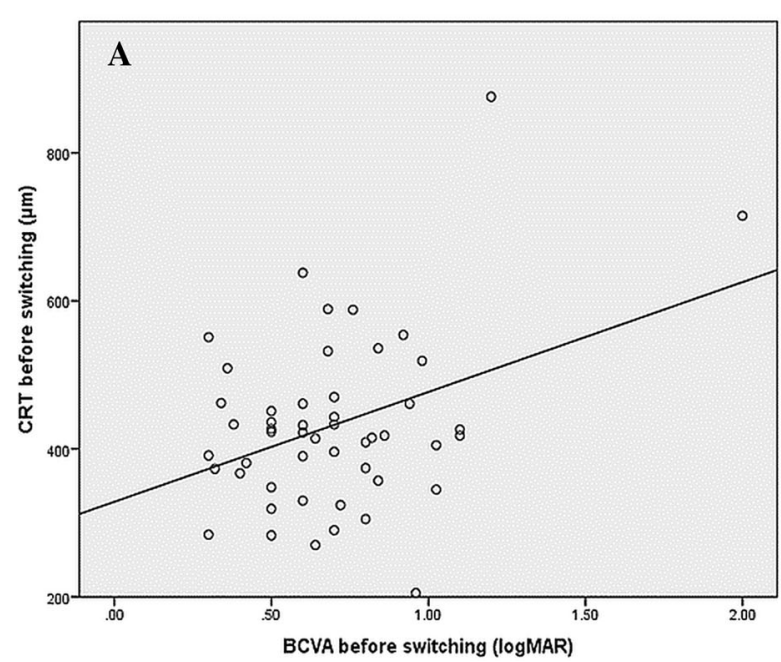

Fig. 2 Correlations between CRT and BCVA at two time points. There was a significant correlation at presentation (a) $\left(r_{\mathrm{p}}=0.969\right)$. However, there was no significant

\section{DISCUSSION}

In this study population, the efficacy of switching anti-VEGF agents is supported in patients with refractory MO secondary to BRVO. Few studies have focused on the use of aflibercept as a second-line agent in non-resolving $\mathrm{MO}$ in BRVO. These include a recent study by Tagami et al. who reported prolongation of the mean intravitreal injection interval after switching treatment from ranibizumab to aflibercept. However, no changes in mean CRT and BCVA were demonstrated after the switch [23]. These findings are further supported by Wirth et al. who reported that switching of treatment not only showed anatomical benefits but also a statistically significant prolongation of the retreatment interval [24]. Sakanishi et al. also reported a short-term beneficial anatomic and functional outcome in patients whose treatment was converted from ranibizumab to aflibercept [22]. This study therefore aims to act as a stepping stone regarding the decision of switching of anti-VEGF therapy, with the primary aim of improving macular integrity and/ or visual acuity.

Parameters such as BCVA and CRT are frequently used to assess/monitor responses to anti-VEGF therapy. This study investigated the

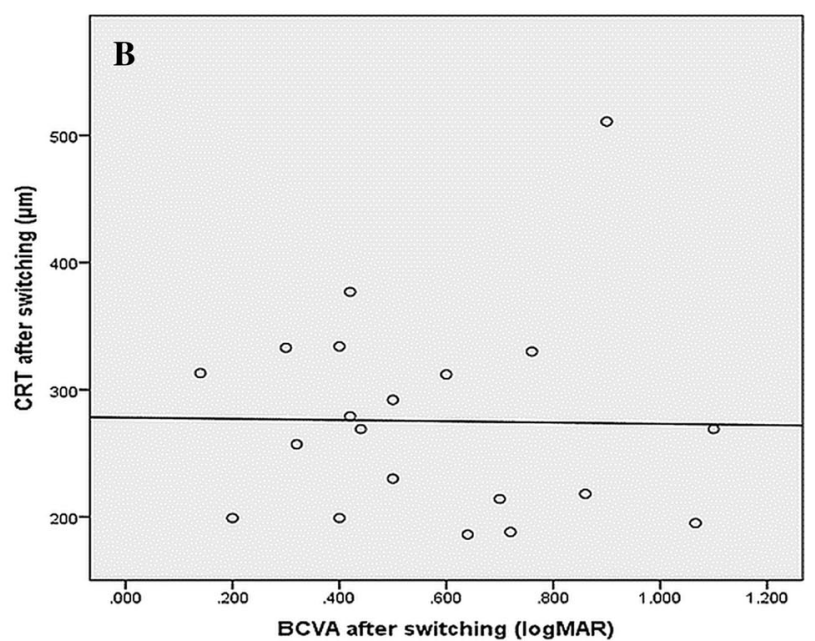

correlation between CRT and BCVA after switching treatment $(\mathbf{b})\left(r_{\mathrm{p}}=-0.17\right)$

benefits of the use of aflibercept therapy in patients with refractory $\mathrm{MO}$ secondary to BRVO, demonstrating a statistically significant improvement in both macular anatomy, as represented by CRT, and BCVA after switching treatment. The study demonstrates resolution of persistent macular oedema (defined as CTR $\leq 300 \mu \mathrm{m}$ ) with mean CRT after switching to aflibercept $275.83 \pm 82.38 \mu \mathrm{m}$. These findings are consistent with the results of the studies by Sakanish et al. and Wirth et al. which reported that eyes with $\mathrm{MO}$ unresponsive to multiple ranibizumab injections demonstrated improvement anatomically after conversion to aflibercept.

However, Mantel at al. in their review of the literature on switching treatment of ARMD highlight that it is difficult to draw accurate conclusions with regards to the efficacy of changing anti-VEGF molecules because of different bias and lack of evidence in improving visual acuity. Aflibercept seems to be more effective in reducing CRT; however, it is not proven to be as effective in increasing visual acuity [25]. Interestingly, Quaranta and Devin [26] presented the outcomes of switching from a fixed dose aflibercept to ranibizumab pro re nata regime. The anatomical and functional outcomes remained stable without increasing 
the number of injections in all but one patient when switching from one drug to another.

The importance of tachyphylaxis has been also investigated by several authors [27-30]. Dirani and Mantel [27] explored the role of tachyphylaxis and the possible correlation to the response to different anti-VEGF drugs in ARMD patients. It has been shown that eyes which developed a drug tolerance are more likely to improve after switching in terms of CRT; however, those results are not maintained over time. The outcome in the long run, particularly with regards to visual acuity, is dependent on several conditions where the history of the disease itself plays a key role. Binder [28] explained that tolerance and tachyphylaxis have been used as synonyms when describing the lack of effect over time of anti-VEGF molecules; however, these terms have different meanings. Pharmacodynamic tolerance means increased expression of VEGF due to increased macrophages, increased expression of VEGF receptors and changes in signal transduction. In order to reduce this effect there are different options, such as increasing the dose, shortening the interval, pausing the treatment, combining different drugs and switching to a different molecule with similar effect. Therefore, the anatomic improvement after switching from ranibizumab to aflibercept may not be due to a more efficient drug, but to a downregulation of antibodies and receptors binding the previous one. A similar beneficial effect is possible if the opposite switching of treatment is made.

Our study further demonstrated a strong correlation of BCVA and CRT at the initial presentation of the cases assessed. This correlation, however, was not shown to be statistically significant after switching treatment to aflibercept, whereby a significant decrease of CRT is observed without an equally significant improvement of BCVA. There are multiple possible causes that could be attributed to these trends. This finding could imply an irreversible damage of the fovea secondary to the presence of persisting intraretinal fluid in the suboptimal responders post first treatment. This proposed theory is supported by the initial observed correlation noted in the study between BCVA and
CRT, but as a result of chronic exposure to the presence of intraretinal fluid, this correlation is no longer sustained. As a result, time elapsing between the actual onset of MO and treatment initiation is noted.

Alongside this considerable decrease of CRT compared to previous ranibizumab treatment, the most clinically significant finding was that MO resolved in all refractory cases. Achieving prompt restoration of macular thickness could have significant benefit for photoreceptors' viability and subsequently patients' central vision [31]. Additional benefits associated with the reduced number of intravitreal injections can have an extensive impact not only on patient care provided but also on service provision by relieving the burden of busy ophthalmology departments.

The correlation noted between CRT at the time of switching and the number of aflibercept injections needed for complete resolution of the MO further implies that more aggressive treatment needs to be considered by clinicians when faced with non-responders after switching from the first anti-VEGF treatment. These findings therefore highlight the urgent need for clinicians to identify suboptimal responders early and switch treatment in order to provide better treatment outcomes anatomically.

Whilst the basis of most studies focusses on improving macular anatomy or visual acuity, it is important to address other non-clinical benefits that can be implied from the data collated. The need for fewer injections with the use of aflibercept therapy in patients with refractory MO secondary to BRVO is duly noted from the data collated from this study. This observation is also supported by the results presented by Tagami et al. and Wirth et al. whereby both studies report significant extension of the interval between treatment episodes when aflibercept is used. This has significant implications on the costs of daily clinical practice as the treatment intervals could be extended without causing detrimental impact to patients. Reducing the number of intravitreal injections as mentioned previously is beneficial not only to patients but also to service providers in the current economical climate. 
It is important to highlight that the present study had certain limitations. The design of the study did not include a control group. Moreover, BCVA and CRT were assessed after switching and for a period of 24 weeks. In terms of the course of disease development and progression, this study was based on a relatively short time period and therefore makes it difficult to extrapolate these findings over a longer time period in order to assess sustainability. Evidently, larger-scale studies need to be conducted to investigate the long-term effects of switching treatment to aflibercept.

Switching anti-VEGF agents has proven to be an efficient strategy in treatment of multiple conditions such as age-related macular degeneration, MO due to central retinal vein occlusion and/or diabetes, with variable results [7-21]. Whilst the overall trends with better outcomes are associated with switching, the potential impact of the use of alternative antiVEGF therapies in managing persisting complications of these eye conditions is limited because of study design. It should be noted that despite previous studies in these other areas studying this switching regime, there is no clear definition to identify so-called responders and non-responders in the study of specific retinal disease. Therefore this study recognises the limitations of this inference and the importance of having a widely accepted definition in order to standardise studies that aim to assess the benefits of switching therapies. A wide consensus regarding definitions of response to treatment and consequently the optimal time for switching from one medication to a different one would play an important role in supporting this mounting evidence of switching therapies in non-responders.

\section{CONCLUSIONS}

Despite the limitations highlighted above, our study provides sufficient evidence demonstrating that switching therapy from intravitreal ranibizumab to aflibercept in this study of patients with persistent MO secondary to BRVO provides improved functional and anatomical outcomes. Aflibercept can therefore be considered to be a clinically effective alternative for patients with BRVO and MO which is suboptimal in response to ranibizumab.

\section{ACKNOWLEDGEMENTS}

The authors would like to thank all the participants of the study.

Funding. No funding or sponsorship was received for this study or publication of this article. The article processing charges were funded by the authors

Authorship. All named authors meet the International Committee of Medical Journal Editors (ICMJE) criteria for authorship for this article, take responsibility for the integrity of the work as a whole, and have given their approval for this version to be published. All authors contributed to the development of the study protocol, the collection of data and statistical analysis. All authors read and approved the final manuscript.

Disclosures. Vasileios E. Konidaris, Konstantinos T. Tsaousis, Rossella Anzidei, Guillermo de la Mata and Alexander J. Brent have nothing to disclose.

Compliance with Ethics Guidelines. Ethics approval was not requested, as the data was collected from an audit on current clinical practice (non-interventional) in the Ophthalmology Department of the University Hospitals of Leicester. All procedures performed in studies involving human participants were in accordance with the ethical standards of the institutional and/or national research committee and with the 1964 Helsinki declaration and its later amendments or comparable ethical standards. Informed consent was obtained from all individual participants included in the study.

Data Availability. The datasets generated during and/or analyzed during the current study are available from the corresponding author on reasonable request. 
Open Access. This article is distributed under the terms of the Creative Commons Attribution-NonCommercial 4.0 International License (http://creativecommons.org/licenses/ by-nc/4.0/), which permits any noncommercial use, distribution, and reproduction in any medium, provided you give appropriate credit to the original author(s) and the source, provide a link to the Creative Commons license, and indicate if changes were made.

\section{REFERENCES}

1. Jaulim A, Ahmed B, Khanam T, Chatziralli IP. Branch retinal vein occlusion: epidemiology, pathogenesis, risk factors, clinical features, diagnosis, and complications. An update of the literature. Retina. 2013;33:901-10.

2. Hahn P, Fekrat S. Best practices for treatment of retinal vein occlusion. Curr Opin Ophthalmol. 2012;23:175-81.

3. NICE. Ranibizumab for treating visual impairment caused by macular oedema secondary to retinal vein occlusion (TA283); 2013. http://www.nice.org. uk/guidance/ta283. Accessed 1 Jan 2018.

4. Campochiaro PA, Heier JS, Feiner L, et al. Ranibizumab for macular edema following branch retinal vein occlusion: six-month primary end point results of a phase III study. Ophthalmology. 2010;117:1102-12.

5. Campochiaro PA, Clark WL, Boyer DS, et al. Intravitreal aflibercept for macular edema following branch retinal vein occlusion: the 24-week results of the VIBRANT study. Ophthalmology. $2015 ; 122: 538-44$.

6. Papadopoulos N, Martin J, Ruan Q, et al. Binding and neutralization of vascular endothelial growth factor (VEGF) and related ligands by VEGF Trap, ranibizumab and bevacizumab. Angiogenesis. 2012;15:171-85.

7. Barthelmes D, Campain A, Nguyen P, et al. Effects of switching from ranibizumab to aflibercept in eyes with exudative age-related macular degeneration. Br J Ophthalmol. 2016;100:1640-5.

8. Amoaku WM, Chakravarthy U, Gale R, et al. Defining response to anti-VEGF therapies in neovascular AMD. Eye. 2015;29:721-31.

9. Ehlken C, Jungmann S, Böhringer D, Agostini HT, Junker B, Pielen A. Switch of anti-VEGF agents is an option for nonresponders in the treatment of AMD. Eye (Lond). 2014;28:538-45.

10. Menke MN, Ebneter A, Zinkernagel MS, Wolf S. Differentiation between good and low-responders to intravitreal ranibizumab for macular edema secondary to retinal vein occlusion. J Ophthalmol. 2016;2016:9875741.

11. Papakostas TD, Lim L, van Zyl T, et al. Intravitreal aflibercept for macular oedema secondary to central retinal vein occlusion in patients with prior treatment with bevacizumab or ranibizumab. Eye (Lond). 2016;30:79-84.

12. Cohen MN, Houston SK, Juhn A, et al. Effect of aflibercept on refractory macular edema associated with central retinal vein occlusion. Can J Ophthalmol. 2016;51:342-7.

13. Konidaris VE, Tsaousis KT, Al-Hubeshy Z, Pieri K, Deane J, Empeslidis T. Clinical real-world results of switching treatment from ranibizumab to aflibercept in patients with diabetic macular oedema. Eye (Lond). 2017;31(11):1629-30.

14. Konidaris V, Al-Hubeshy Z, Tsaousis KT, Gorgoli K, Banerjee S, Empeslidis T. Outcomes of switching treatment to aflibercept in patients with macular oedema secondary to central retinal vein occlusion refractory to ranibizumab. Int Ophthalmol. 2018;38(1):207-13.

15. Lim LS, Ng WY, Mathur R, et al. Conversion to aflibercept for diabetic macular edema unresponsive to ranibizumab or bevacizumab. Clin Ophthalmol. 2015;16:1715-8.

16. Wood EH, Karth PA, Moshfeghi DM, Leng T. Shortterm outcomes of aflibercept therapy for diabetic macular edema in patients with incomplete response to ranibizumab and/or bevacizumab. Ophthalmic Surg Lasers Imaging Retina. 2015;46:950-4.

17. Shah CP, Heier JS. Aflibercept for diabetic macular edema in eyes previously treated with ranibizumab and/or bevacizumab may further improve macular thickness. Ophthalmic Surg Lasers Imaging Retina. 2016;47:836-9.

18. Lee JH, Lee WK, Kim SE. Short-term outcomes of switching to ranibizumab therapy for diabetic macular edema in patients with persistent fluid after bevacizumab therapy. J Ocul Pharmacol Ther. 2016;32:659-64.

19. Hussain RM, Ciulla TA. Treatment strategies for refractory diabetic macular edema: switching antiVEGF treatments, adopting corticosteroid-based treatments, and combination therapy. Expert Opin Biol Ther. 2016;16:365-74. 
20. Hanhart J, Chowers I. Evaluation of the response to ranibizumab therapy following bevacizumab treatment failure in eyes with diabetic macular edema. Case Rep Ophthalmol. 2015;6:44-50.

21. Katz G, Moisseiev E, Goldenberg D, et al. Ranibizumab for persistent diabetic macular edema after bevacizumab treatment. Eur J Ophthalmol. 2017;27(2):210-4.

22. Sakanishi Y, Usui-Ouchi A, Tamaki K, Mashimo K, Ito R, Ebihara N. Short-term outcomes in patients with branch retinal vein occlusion who received intravitreal aflibercept with or without intravitreal ranibizumab. Clin Ophthalmol. 2017;11:829-34.

23. Tagami M, Sai R, Fukuda M, Azumi A. Prolongation of injection interval after switching therapy from ranibizumab to aflibercept in Japanese patients with macular edema secondary to branch retinal vein occlusion. Clin Ophthalmol. 2017;11:403-8.

24. Wirth MA, Becker MD, Graf N, Michels S. Aflibercept in branch retinal vein occlusion as second line therapy: clinical outcome 12 months after changing treatment from bevacizumab/ranibizumab-a pilot study. Int J Retina Vitreous. 2016;2:20.

25. Mantel I, Gillies MC, Souied EH. Switching between ranibizumab and aflibercept for the treatment of neovascular age-related macular degeneration. Surv Ophthalmol. 2018;63:638-45.

26. Quaranta M, Devin F. Switch from aflibercept to ranibizumab: up to 2 years follow-up post View2 study. IOVS. 2014;55:3935.

27. Dirani A, Mantel I. Ranibizumab treatment history as predictor of the switch-response to aflibercept: evidence for drug tolerance. Clin Ophthalmol. 2018;12:593-600.

28. Binder S. Loss of reactivity in intravitreal antiVEGF therapy: tachyphylaxis or tolerance? $\mathrm{Br} \mathrm{J}$ Ophthalmol. 2012;96:1-2.

29. Eghøj MS, Sørensen TL. Tachyphylaxis during treatment of exudative age-related macular degeneration with ranibizumab. $\mathrm{Br} \mathrm{J}$ Ophthalmol. 2012;96:21-3.

30. Zhang M. Attention to the non-responders to antiVEGF treatment of wet age-related macular disease. Zhonghua Yan Ke Za Zhi. 2014;50:406-10.

31. Mori Y, Suzuma K, Uji A, et al. Restoration of foveal photoreceptors after intravitreal ranibizumab injections for diabetic macular edema. Sci Rep. 2016;6:39161. 